\title{
A PROPOS DE TROIS CARACTÈRES INTÉRESSANT LA TAXONOMIE DES TROMBICULIDE (ACARINA)
}

\section{Par P.-H. VERCAM MEN-GRANDJEAN}

Trois caractères taxonomiques fondamentaux jouent un rôle prépondérant dans la systématique des TROMBICULID $\approx$.

Deux de ces caractères ont trait à l'état larvaire, le troisième concerne la nymphe.

A. - Les fémurs des pattes peuvent être d'une seule pièce, ou segmentés en basi- et télo-fémurs.

B. - Les Iames chélicérales peuvent être armées de une ou plusieurs dents sur la face dorsale ou être sans dent.

C. - Les nymphes des GAHRLIEPIIN.E portent, sur la face dorsale du tarse I, un éperon subapical typique.

Des découvertes récentes ont fait connaitre certaines espèces « paradoxales » qui mettent en défaut un système de classification parfait jusqu'alors.

\section{A. - De l'unité et de la division fémorale}

L'unité ou la division des fémurs, chez les TRombiculids: larvaires, a donné naissance à la « formule segmentaire des pattes ». Cette formule concise et objective fut préconisée, dès 1947, par Wharton et acceptée d'emblée (1). Cet auteur établit ainsi une sćrie de règles traduites par le tableau suivant:

7.7.7. = Trombiculinæ Ewing $1946+$ Apoloniinze Wharton 1947.

7.6.6. = Fahrliepiinæ Womersley 1952 (= Walchiinæ Ewing 1946).

6.6.6. = Leeuwenhoekiina Womersley 1944 .

Or, il advient qu'en 1951 Lipovsky trouve un Ascoschöngastia qui présente la formule 7.6.6. Il crée, en conséquence, un genre nouveau : Pseudoschöngastia, qu'il range dans la sous-famille des WALCHIINA (2).

AnÑ. de Parasitologie, t. XXXI, N ${ }^{\circ} 4 .-1956$. 


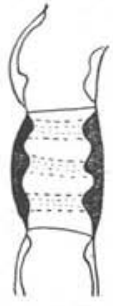

A

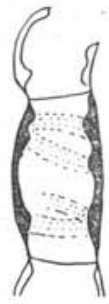

B

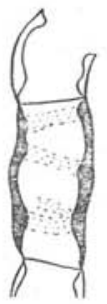

C

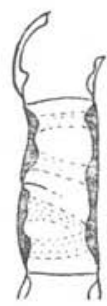

D

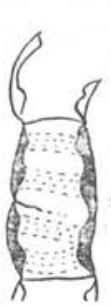

E

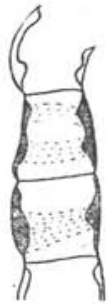

$\mathrm{F}$

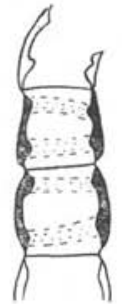

G
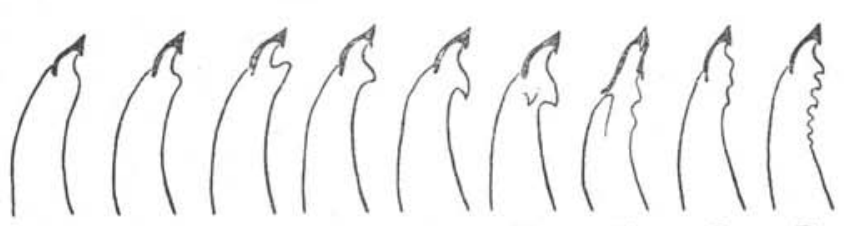

$P$

E

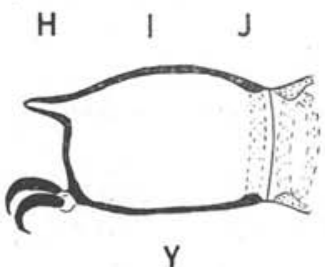

K

N

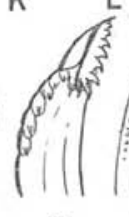

$\mathrm{x}$

W

M

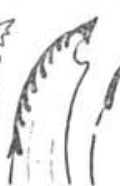

V

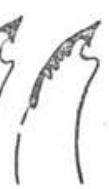

$\mathrm{U}$

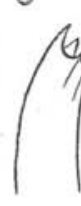

T

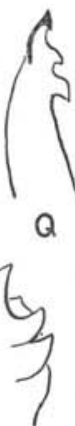

$\mathrm{S}$

函

F1G. - 1) de A à $\mathrm{G}$ : divers états du fémur postérieur.

A. Leeuwenhoekiinw, Acomatacarus (Hyracarus) sp.

B. GAHRLIEPIINA et certains Schoutedenichia sp. et Trombicula sp.

C. Trombicula du groupe « verrucascuta».

D. Fémur « hémi-soudé » de certains « verruscula».

E. - - de certains Schoutedenichia.

$F$ et $G$. Deux types de fémurs segrmentés en basi- et télofémur.

2) de $\mathrm{H}$ à $\mathrm{X}$ : divers schémas de chélicères.

H, I et K. Trombicula, Euschöngastia et divers GAHRLIEPIIN E.

J. Certains Trombicula (Neotrombicula, Blankaartia, Heaslipia), Neoschöngastia.

L, M, N, O. Schoutedenichia, Fainiella, Giroudia longiscutullata, Doloisia.

P. Schöngastia, Neoschöngastia.

Q. Endotrombicula, Phrynacarus.

R. Radfordiana, Schöngastia.

S. Myotrombicula.

T. Oenoschöngastia.

U. Giroudia brennani.

V, W, X. Divers Acomatacarus (LEEUWENHOEKIINAE).

3) Y, tarse antérieur d'une nymphe de GAHRLIEPIINA et de Schoutedenichia. 
L'année suivante, c'est Fuller qui inaugure le genre Walchiella dans la même sous-famille des WALCHIINA, à cause de la formule segmentaire des pattes de Trombicula oudemansi Walch 1922 (3) (4) (5).

En 1953, Jadin et Vercammen-Grandjean instaurent le genre Schoutedenichia à l'occasion de deux espèces qu'ils classent ainsi dans la sous-famille des GAHRLIEPIINAE. Pourtant, mise à part leur formule segmentaire 7.6.6., ces espèces sont bien plutôt des Euschöngastia (6) (7).

Brennan crée, entre-temps, le genre Anomalaspis pour une espèce qui, peut-être, se rapproche de Tecomatlana Hoffman 1947, mais dont la formule est 7.6.6. (8) (9).

En 1952, déjà, Womersley prend position en affirmant sa conviction de la faible valeur taxonomique de l'unité ou de la segmentation fémorale du point de vue sub-familial (10). Il s'appuie sur les cas de certaines espèces en tous points voisines, sauf en ce qui concerne la formule segmentaire des pattes, et qui, pour cette dernière raison, se voyent impitoyablement séparées et cataloguées dans des familles ou des sous-familles différentes.

Plus récemment, Audy récolte un Trombicula incontestable, dont la formule est 7.6.6. Il prend position fermement en appelant Trombicula normi cette larve dont la place est tout indiquée à côté de $T$. minor (11).

Vers la même époque, Varma trouve un Trombicula de fort petite taille, mais dont la formule est, cette fois, 6.6.6. Trombicula krishani est classé convenablement et non parmi les Leeuwenhoekiinx où une application rigide de la systématique en vigueur aurait pu le mener (12).

J'ai eu la bonne fortune de pouvoir étudier un lot important de TROMBICULIDA: larvaires qui comptent onze espèces nouvelles. Toutes appartiennent à un même groupe homogène dans le genre Trombicula. Ce groupe, qui a été appelé «Verruscuta», est en voie de publication. Certaines espèces ont déjà été décrites, telles que : T. batui Philip \& Traub, 1950, T. panieri et T. rodhaini Jadin \& Vercammen-Grandjean, 1952 et $T$. jadini Vercammen-Grandjean, 1952 (13) (14). Le total actuel des espèces de ce groupe atteint dix-huit.

On peut dire à leur sujet :

$x^{\circ}$ que sur l'ensemble des spécimens étudiés, les formules segmentaires des pattes observées furent les plus diverses; 
$2^{\circ}$ qu'il existe des fémurs non intégralement segmentés. Par écrasement mitigé des préparations, ces états d' « hémisoudures \& furent parfaitement mis en évidence. Conventionnellement, cette segmentation incomplète sera indiquée par « 6 ! », dans la formule segmentaire des pattes. Les combinaisons suivantes furent observées: 6!6.6., 7.6.6., 7.6!6., 7.6!6!, 7.7.6., 7.7.6!, et 7.7.7. ;

$3^{\circ}$ que, dans la même espèce, on peut rencontrer des formules voisines de celle qui domine;

$4^{\circ}$ que la segmentation semble être en fonction directe de la taille ; autrement dit, que les espèces menues ont une formule qui tend vers 6.6.6., tandis que les espèces plus grandes tendent vers 7.7.7..

Il est impossible, dans le cadre de cette note, de s'étendre sur les circonstances probables de semblables variations. Quelles qu'en soient d'ailleurs les origines, c'est leur application en matière de systématique qui nous importe.

\section{B. - De la denticulation chélicérale}

En 1946, Ewing se base sur la multidenticulation des chélicères, d'une part, et la non ou l'unidenticulation d'autre part, pour séparer en deux genres distincts des espèces jusqu'alors réunies (15). Ce système sert, dès lors, de base dans maintes différenciations d'ordre générique.

En 1952, Jadin et Vercammen-Grandjean s'en servent pour décrire leur nouveau genre Giroudia en marge de Gateria Ewing 1938 (13). Je m'en sers pour un parasite nasicole voisin du genre Walchia Ewing 1931, que j'appelle : Fainiella (7).

Il semble pourtant que ce caractère, tout comme celui qui précède, ait été utilisé de façon trop rigoureuse. Et, par exemple, des espèces rangées dans le genre Schöngastia Oudemans 1910 y sont déplacées, telles sont : S. oudemansi (Walch 1923), S. bidentata Womersley 1952 et $S$. oculicola Wom. 1952.

Par contre, Euschöngastia edwardsi (Gunther 1939) et E. capensis Lawrence 1952 sont de véritables Schöngastia, dont ils possèdent le « faciès » typique, à défaut de la multidenticulation chélicérale.

\section{C. - De l'éperon tarsal des nymphes de GAHRLIEPIINAE}

En 1952, Womersley définit les GAHRLIEPIINA, nymphes et adultes, par la seule présence d'un éperon subapical sur le dos du tarse I (10). 
Or, en 1953, Jadin et Vercammen-Grandjean trouvent, presque simultanément, trois Euschöngastia dont les nymphes d'élevage sont dûment armées de l'éperon tarsal I (16) (17).

Cette découverte pourrait faire croire à la caducité du caractère. Au lieu de cela, elle raffermit la thèse de Womersley, tout en nous apprenant l'existence de Trombiculidx intermédiaires entre les deux sous-familles: TROMBICULINA: et GAHRLIEPIINA.

\section{Conclusions}

A - 1) La formule segmentaire des pattes, 7.6.6., est constante - et donc valable - pour tous les GAHRLIEPIINE, dont elle peut continuer à être considérée comme un caractère phylogénique d'ordre sub-familial.

2) La formule 7.7.7. est caduque pour la sous-famille des TROMBICULINE. En corollaire, il y a lieu de ramener au sein de cette dernière sous-famille les espèces qui furent indûment placées parmi les GAHRLIEPIINA et qu'une grande majorité d'autres caractères apparentent aux TROMBICULINA, c'est-à-dire : Pseudoschöngastia, Walchiella, Anomalaspis et Schoutedenichia.

B - 1) La denticulation chélicérale, - nulle, unique ou multiple -, de disposition, de forme ou de grandeur variable, est un caractère de valeur générique inégale.

2) La diversité des cas observés plaide en faveur de l'hypothèse de modifications sous l'influence de facteurs extérieurs. Autrement dit, il semblerait que le mode de vie et genre de parasitisme d'une espèce donnée soient déterminants quant à l'armature chélicérale.

3) Dans les cas de Giroudia Jadin \& Vercammen-Grandjean, 1952 et de Fainiella Vercammen-Grandjean, 1953, le statut de sous-genres, respectivement de Gateria Ewing 1938 et de Walchia Ewing 1931, s'impose.

4) Les espèces Schöngastia oudemansi (Walch 1922), S. bidentata Womersley 1952 et $S$. oculicola Womersley 1952 doivent être reclassées dans les Euschöngastia.

5) Inversement, les Euschöngastia edwardsi (Gunther 1939) et E. capensis (Lawrence 1949) sont d'authentiques Schöngastia. 
C - 1) L'éperon tarsal des nymphes reste un caractère général propre aux GAHRLIEPIINAE.

2) Il est, en outre, l'apanage des nymphes de certains TROMBICULIDE dont les larves possèdent des caractères également partagés entre TROMBICULINE et GAHRLIEPIINE. Le nombre existant actuellement de ces espèces, que nous grouperons sous le nom de Schoutedenichia, est suffisamment éloquent : environ 35 pour l'Afrique et la région Asiatique-Pacifique (18).

Je remercie bien sincèrement MM. J. R. Audy, J. M. Brennan, H. S. Fuller, J. B. Jadin, C. D. Radford et H. Womersley, grâce à l'aide et aux conseils desquels la présente ètude fut rendue possible.

\section{Laboratoire médical provincial de Kivu, Section: Biologie. Bukavu, le 10 février 1956.}

\section{Bibliographie ET RÉFÉrEnces}

(1) Wharton (G. W.). - 1947. Studies on North American Chiggers. II. The sub-families and Womersia strandtmanni n. g., n. sp. J. Parasitol., 33, $4,380-384$.

(2) Lipovsky (L. J.). - 1951. A new genus of Walchiina. J. Kans. Ent. Soc., 24, 95-102.

(3) Whartox (G. W.) et Fullen (H. S.). - 1952. A Manual of the Chiggers. Ent. Soc. Washing., 4, 95.

(4) Fuller (H. S.). - 1952. The Mite larve of the family Trombiculide in the Oudemans collection. Zool. Verhandel., 18, 220-227.

(5) Walch (E.). - 1922. In Geneesk. Tijdschr. Ned. Ind., 62, 5, 563-564.

(6) Jadin (J.-B.) et Vercammen-Ginandean (P.-H.). - 1954. Cinq nouvelles espèces de Trombiculidxe du Piuanda-Urundi et création du genre nouveau : Schoutedenichia. Ann. Mus, Congo, Terv., Zool., I, 194-206.

(7) Vercammen-Grandjean (P.-H.). - 1953. Un nouveau biotope parasitaire des Trombiculide larvaires chez les rongeurs du Ruanda-Urundi. Rev. Zool. Bot. Afr., 47, 1-2, 17-29.

(8) Brennan (J. M.). - 1952. Two new venezuelan Chiggers. J. Parasitol., 38, 2, 143-146.

(9) Hoffmann (A. M.). - 1947. Un nuevo genero de Trombidido Mexicano. Ann. Escula Nac. Cien. Biol., 4, 451-457.

(10) Womerslex (H.). - 1952. The scrub-typhus \& scrub-itch Mites of the Asiatic-Pacific Region. Rec. Sth. Austr. Mus., Adelaïde, 10.

(11) Audy (J. R.). - 1954, in litt.

(12) Audy (J. R.). - 1954, in litt. 
(13) Philip (C. B.) et Traub (R.). - 1950. Two new species or Trombiculid Mites from Malayan bats. J. Parasitol., 36, 29-33.

(14) Jadin (J.-B.) et Vercammen-Grandjean (P.-H.). - 1952. Les Trombiculide larvaires du Ruanda-Urundi. Ann. Soc. Bel. Méd. Trop., 32, 1, 593-656.

(15) Ewing (H. E.). - 1946. Notes on the Taxonomy of three genera of Trombiculid Mites, together with the description of a new genus. Proc. Biol. Soc., Washingt., 59, 69-72.

(16) Jadin (J.-B.) et Vercammen-Grandjean (P.-H.). - 1954. Deux Trombiculide larvaires parasites hypodermes de certains rongeurs. Rev. Zool. Bot. Afr., 49, 283-292.

(17) Jadin (J.-B.), Vercammen-Grandjean (P.-H.) et Herman (F.). - 1954. Un Trombiculide nasicole nouveau. Rev. Zool. Bot. Afr., 49, 273-282.

(18) Vercammen-Grandjean (P.-H.) et Audy (J.-R.). - 1956. Note concernant la Taxonomie des Trombiculide (Acarina) avec, comme corollaire, la révision et l'élargissement du genre Schoutedenichia Jad. et Ver. 1954, in press. 